\title{
A TWO-DIMENSIONAL CARTESIAN AND AXISYMMETRIC STUDY OF COMBUSTION-ACOUSTIC INTERACTION ${ }^{1}$
}

\author{
Caroline Hood ${ }^{2}$ and Abdelkader Frendi ${ }^{3}$ \\ University of Alabama in Huntsville \\ Huntsville, AL-35899
}

\begin{abstract}
This paper describes a study of a lean premixed (LP) methane-air combustion wave in a two-dimensional Cartesian and axisymmetric coordinate system. Lean premixed combustors provide low emission and high efficiency; however, they are susceptible to combustion instabilities. The present study focuses on the behavior of the flame as it interacts with an external acoustic disturbance. It was found that the flame oscillations increase as the disturbance amplitude is increased. Furthermore, when the frequency of the disturbance is at resonance with a chamber frequency, the instabilities increase. For the axisymmetric geometry, the flame is found to be more unstable compared to the Cartesian case. In some cases, these instabilities were severe and led to flame extinction. In the axisymmetric case, several passive control devices were tested to assess their effectiveness. It is found that an acoustic cavity is better able at controlling the pressure fluctuations in the chamber.
\end{abstract}

\section{Introduction}

Environmental awareness leads to increased demands for the ultra-low emission engines. Lean premixed (LP) combustion is the most effective process for lowering the emission. As a result, the research and development of gas turbines, which use LP combustors, have been a major interest for the past two decades. Future gas turbine engines must provide ultra-low emissions and high efficiency at low cost while maintaining the reliability and operability of present day engines. The demands for increased performance and decreased emissions have resulted in advanced combustor designs that are critically dependent on efficient fuel/air mixing and lean operation. However, all combustors, but most notably lean-burning low-emissions combustors, are susceptible to combustion instabilities. These instabilities typically occur due to sustained pressure perturbations of acoustic nature in a chamber where combustion takes place. They arise when different chamber processes couple in such a way that inherent disturbances in the system are self-excited via interactions with the combustion process.

\footnotetext{
${ }^{1}$ This material is a work of the U.S. Government and is not subject to copyright protection in the United States.

${ }^{2}$ Graduate Research Assistant, Member of AIAA.

${ }^{3}$ Professor of Mechanical and Aerospace Engineering, Senior Member of AIAA.
} 
The acoustic pressure alters the flame, which in turn drives sound by producing unsteady heat release.

Frendi [1] concentrated on the coupling between the unsteady heat release rate from combustion and the acoustic energy of the combustion chamber. Furthermore, he also studied the effect of adding a Helmholtz resonator to the system. Similarly, Paxson and Quinn [2] performed a comprehensive study of the effect of pressure waves on the acoustic modes of the combustion chamber and how it instigates various forms of instabilities. In addition, Paxson [3] studied the effect of abrupt change of area in a lean premixed combustor rig and how this change of area expedites the phenomena of combustion instabilities. On the other hand, Prasad [4] investigated the effect of external pressure perturbations on "premixed flames" using detailed chemistry. His results indicated that reflected and transmitted waves differed significantly from incident waves. However, Wu, et. al. [5] studied the flame structure and its associated hydrodynamic field in detail. They proposed that combustion instability arises when acoustic modes of the chamber are excited and amplified by the flame instabilities through mutual resonance.

In the present study, a two-dimensional Cartesian and axisymmetric model is used to study the effects acoustic coupling on combustion instabilities. A one-step global reaction is used to model the chemical kinetics.

\section{Mathematical Model}

The Mathematical model consist of the nonlinear Navier-Stokes equations with a one-step irreversible exothermic chemical reaction of the form

$$
v_{F}(\text { Fuel })+v_{o}(\text { Air }) \rightarrow v_{P} \text { (Products) }
$$

where Fuel and Air are assumed to be in gas phase.

A set of unsteady, compressible Navier-Stokes equations for a two-dimensional axisymmetric system used to describe our gas-phase reacting flow problem, is as follows

$$
\frac{\partial Q}{\partial t}+\frac{\partial F}{\partial x}+\frac{\partial G}{\partial r}=B
$$

where $Q$ is the state vector, $F$ and $G$ are the fluxes in the $x$ and $r$ directions, respectively, and $B$ is the source term that arises in cylindrical coordinates in addition to the reacting flow.

$$
Q=r\left[\begin{array}{c}
\rho \\
\rho u \\
\rho v \\
\rho e \\
\rho Y_{F} \\
\rho Y_{o}
\end{array}\right] \quad F=r\left[\begin{array}{c}
\rho u \\
\rho u^{2}-\tau_{x x}+p \\
\rho u v-\tau_{x r} \\
u(\rho e+p)-\lambda \frac{\partial T}{\partial x} \\
\rho u Y_{F}-\rho D \frac{\partial Y_{F}}{\partial x} \\
\rho u Y_{o}-\rho D \frac{\partial Y_{o}}{\partial x}
\end{array}\right] \quad G=r\left[\begin{array}{c}
\rho v \\
\rho u v-\tau_{x r} \\
\rho v^{2}-\tau_{r r}+p \\
v(\rho e+p)-\lambda \frac{\partial T}{\partial r} \\
\rho v Y_{F}-\rho D \frac{\partial Y_{F}}{\partial r} \\
\rho v Y_{o}-\rho D \frac{\partial Y_{O}}{\partial r}
\end{array}\right] \quad B=\left[\begin{array}{c}
0 \\
0 \\
p-\tau_{\theta \theta} \\
r q_{c o m b}{ }^{\prime \prime}+r q_{i g}{ }^{\prime \prime} \\
r m_{F}{ }^{\prime \prime} \\
r m_{O}{ }^{\prime \prime \prime}
\end{array}\right]
$$


where, $Y_{O}$ and $Y_{F}$ are Oxygen and fuel mass fractions, respectively. The source terms in the energy equations are given by

$$
\begin{gathered}
\dot{q}_{c o m b}^{m}=h_{c}\left(\rho Y_{F}\right)^{m}\left(\rho Y_{o}\right)^{n} A \exp \left(\frac{-E_{a}}{R T}\right) \\
\dot{q}_{i g}^{m}=\left[H(\tau)-H\left(\tau-\tau_{i g}\right)\right] \dot{E}_{i g}^{m} \exp \left[-g(P)\left(\left(\frac{x-x_{0}}{r_{i g}}\right)^{p}+\left(\frac{r-r_{0}}{r_{i g}}\right)^{p}\right)\right] \\
\dot{m}_{F}^{m}=-v_{F} M_{F} \frac{\left(\rho Y_{F}\right)^{m}\left(\rho Y_{o}\right)^{n}}{\left(M_{F}\right)^{m}\left(M_{O}\right)^{n}} A_{r} \exp \left(\frac{-E_{a}}{R T}\right) \\
\dot{m}_{O}^{m \prime \prime}=-v_{O} M_{o} \frac{\left(\rho Y_{F}\right)^{m}\left(\rho Y_{o}\right)^{n}}{\left(M_{F}\right)^{m}\left(M_{O}\right)^{n}} A_{r} \exp \left(\frac{-E_{a}}{R T}\right)
\end{gathered}
$$

\section{Results and Discussions}

The System of equations given by (2) - (3) with source terms (4) - (7) is solved using the Gottlieb-Turkel scheme [6], which is second order accurate in time and fourth order accurate in space. This scheme is based on MacCormack second order accurate Scheme. Along with Gottlieb-Turkel scheme, fourth order artificial dissipation terms given by Anthony Jameson [7] were also explicitly added to capture sudden discontinuities. To solve the above system of equations, coding is done in Matlab. A domain having of $6 \mathrm{~cm}$ in each direction is used with $200 \times 200$ grid points. At first the acoustic modes of the 2D Cartesian as well as axisymmetric chamber are determined using a numerical bomb test. This is achieved by using the source term given by equation (5). In this numerical experiment, the combustion source terms are ignored. The modes of the chamber are then obtained by taking the FFT of the time history of the pressure time history. To validate our work, a special case was studied using an axisymmetric chamber with a diameter and length of 2.125 and 8.5 inches, respectively. The new chamber geometry corresponds to the chamber used by Moser et al. [8] in their experiments.

\section{Validation Case:}

Figure 1 shows the temperature contours in the axisymmetric chamber shortly after ignition. A symmetrical high temperature kernel is shown at the center of the chamber. Figure 2 shows the corresponding instantaneous pressure contours. The contours show clearly the presence of a longitudinal mode. This is confirmed by the time history and the 
corresponding power spectral density of Figure ( $3 a \& b)$ showing a strong mode at around $3000 \mathrm{~Hz}$. These results agree well with those obtained experimentally by Moser et al. [8], Fig (3c), though our frequency is slightly higher because of a small difference in the chamber dimensions.

\section{Combustion-Acoustic Interaction Results:}

Figures (4)a-b and (5)a-b show the pressure time history at the center of the chamber and the corresponding FFT for both the Cartesian (Fig.4(a)-(b)) and axisymmetric (Fig. 5(a)(b)) geometries, respectively. The pressure signature shown in figures (4) and (5) is obtained from a numerical bomb experiment. The time histories show the presence of several frequencies which is confirmed by the power spectral densities. The corresponding temperature history at the center of the Cartesian chambers are shown on Fig. (6). The figure shows a transient behavior of the temperature. The instantaneous temperature contours corresponding to the Cartesian geometry is shown on Fig. (7) for the case without acoustic disturbance. From Fig.(7), it is observed that a symmetric kernel is obtained. When an acoustic disturbance is interacting with the combustion wave, depending upon the amplitude and the frequency of the disturbance, it is found that the flame is strongly affected by the acoustic disturbance as shown on Fig. (8) and (9).

\section{Passive Control}

Figure 10 shows a composite plot of the grid used, the pressure contours, the time history of the pressure and the corresponding power spectral density for an axisymmetric combustion chamber without a passive device. A strong fundamental and harmonic are shown on the power spectral density. When a quarter-wave is added to the chamber, Figure 11 shows that the pressure fluctuations are significantly reduced and the power spectrum shows a large number of peaks with a weakened fundamental and harmonic. Figure 12 shows the effect of using an acoustic cavity on the resonant interaction. Further reduction in the amplitude of the pressure fluctuations is obtained. Both passive control devices act to detune the chamber.

\section{Conclusions}

The results show that acoustic-combustion coupling plays an important role in combustion instability. The response of a premixed flame as it interacts with an externally imposed acoustic disturbance were studied. Based on the results presented, the following conclusions are made:

1. The instabilities of the flame increase by increasing the disturbance amplitude.

2. When the frequency of the disturbance is at resonance with the chamber frequency, the instabilities increase and lead to flame extinction.

3. For the axisymmetric geometry, the flame behaves more unstably compared to the one for the Cartesian system. 
4. An acoustic cavity is a more effective passive control device than either a baffle or quarter wave.

Comparisons to experimental results show reasonable agreement.

\section{Acknowledgements}

This work was supported by grant NCC8-200 from NASA Marshall Space Flight Center with Tom Nesman as the technical monitor.

\section{References}

[1] Frendi, A., "On the role of Acoustic Coupling on Combustion Instabilities," AIAA2003-3181, Hilton Head, South Carolina, 2003

[2] Quinn, D., and Paxson, D., "A Simplified Model for the.Investigation of Acoustically Driven Combustion Instabilities," Cleveland, OH. AIAA-98-3764

[3] Paxson, D., "A Sectored One-Dimensional Model for Simulating Combustion Instabilities in Premix Combustors," Glenn Research Center. Cleveland, Ohio, 2000. AIAA-2000-0313.

[4] Prasad, K., "Interaction of Pressure Perturbations with Premixed Flames," Combustion and Flame, Vol. 97, 1994, pp.173-200.

[5] Wu, X., Wang, M., and Moin, P., "Combustion instability due to the nonlinear interaction between sound and flame," Center for Turbulence Research, Annual Research Briefs 2001.

[6] Gottlieb, D., and Turkel, E., "Dissipative Two-Four Methods for Time-Dependent Problems," 1976, Mathematics of Computations, Vol. 30, No. 136, 703-723.

[7] Jameson, A., Schmidt, W., and Turkel, E., "Numerical Solutions of the Euler Equations by Finite Volume Methods Using Runga-Kutta Time-Stepping Schemes," 1981, AIAA-81-1259.

[8] Moser, M. and Hall, T., CUIP quarterly report, June 2006. 


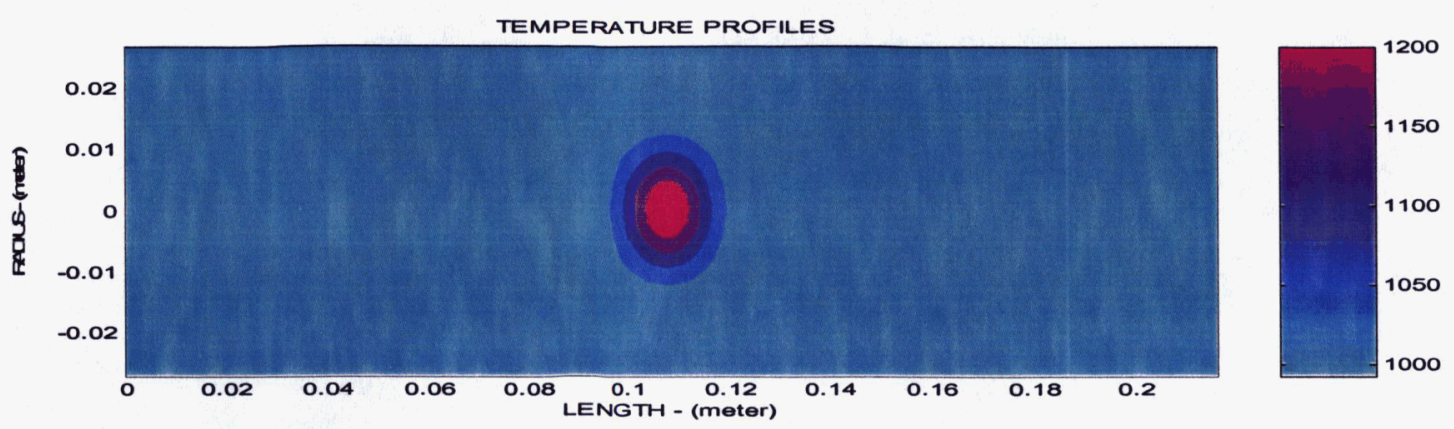

Figure 1: Instantaneous temperature contours of a premixed-flame in a 2-D axisymmetric chamber at $\mathrm{t}=0.02$ seconds.

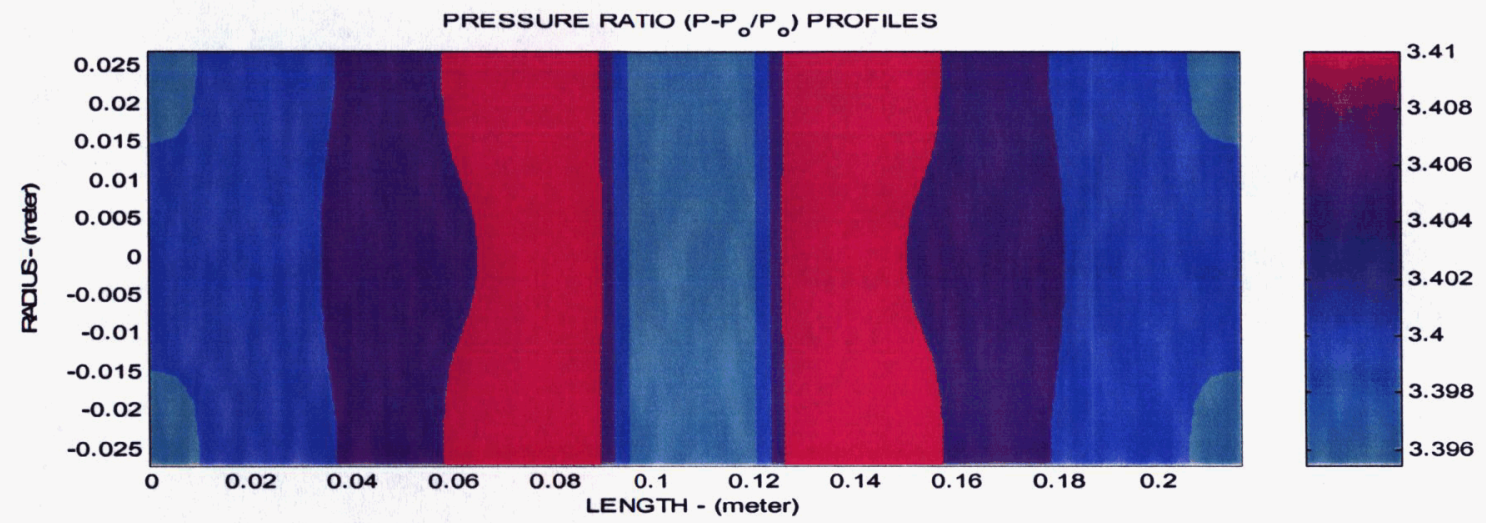

Figure 2: Instantaneous pressure contours of a premixed-flame in a 2-D axisymmetric chamber at $\mathrm{t}=0.02$ seconds.

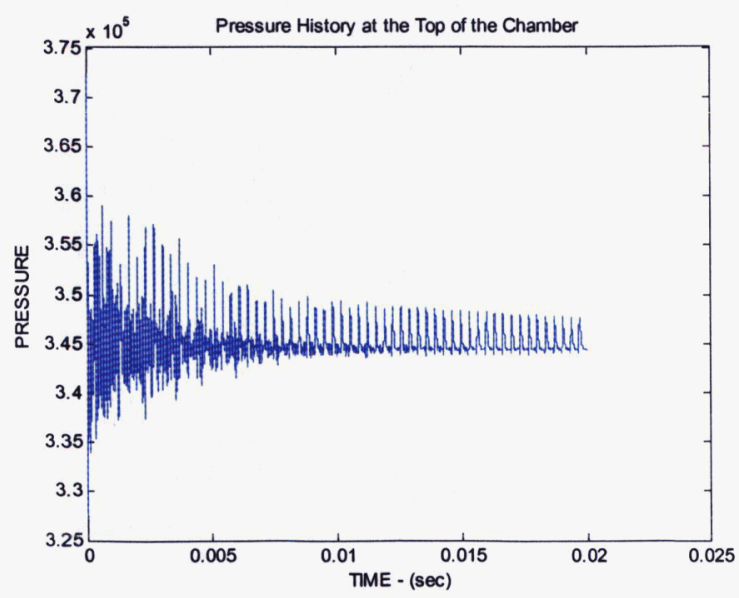

(a)

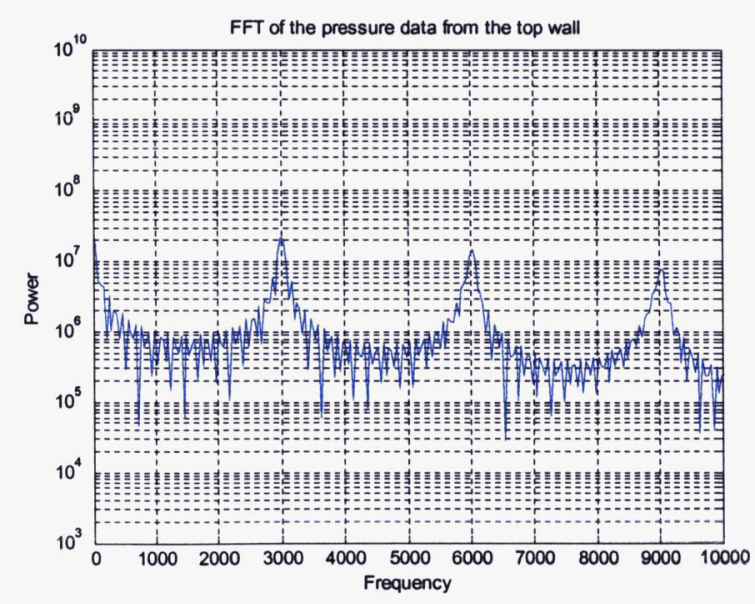

(b) 


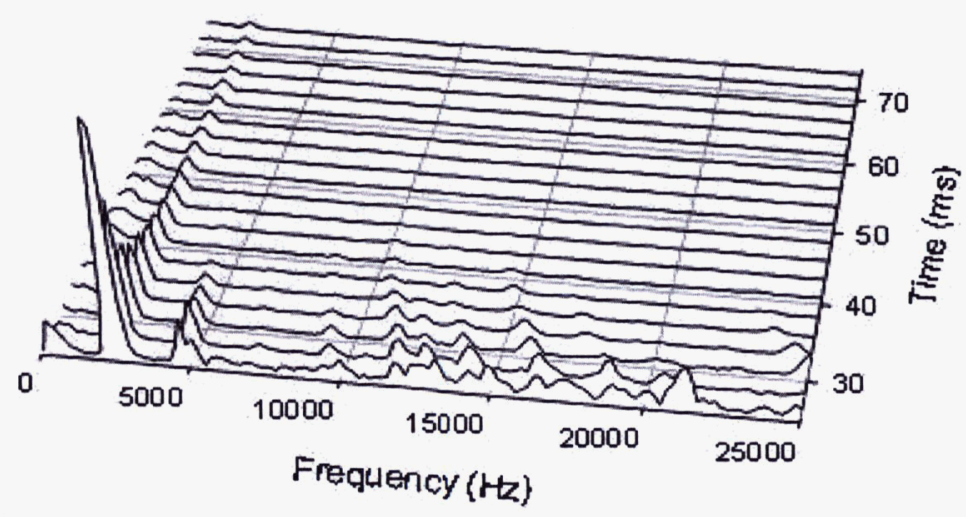

(c)

Figure 3: (a) Time history of the pressure of a lean-premixed flame in a 2-D axisymmetric geometry, (b) the FFT of the corresponding pressure data for the special case and (c) The frequency vs. time plot from the experiment results by Moser [8]

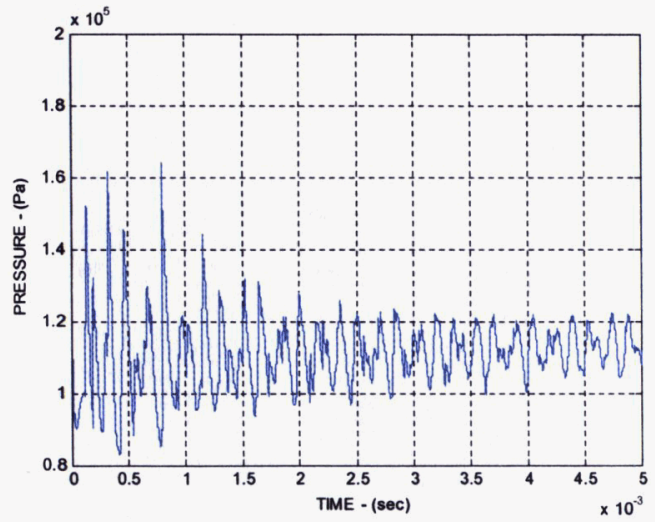

(a)

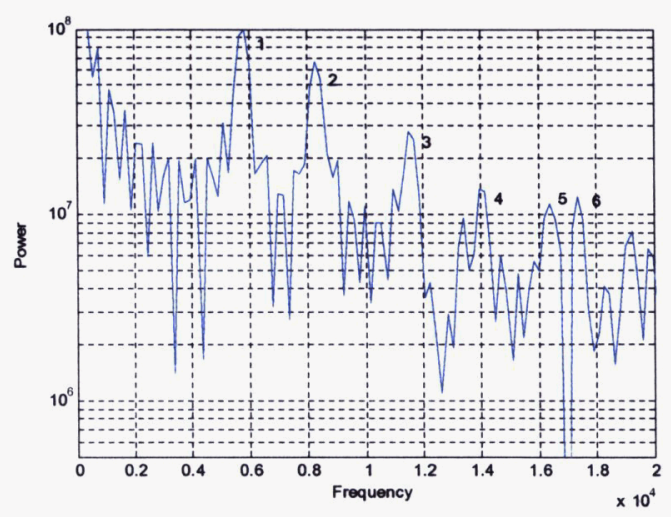

(b)

Figure 4: (a) Time history of the pressure of a lean-premixed flame in a two-dimensional Cartesian geometry and (b) the FFT of the corresponding pressure data

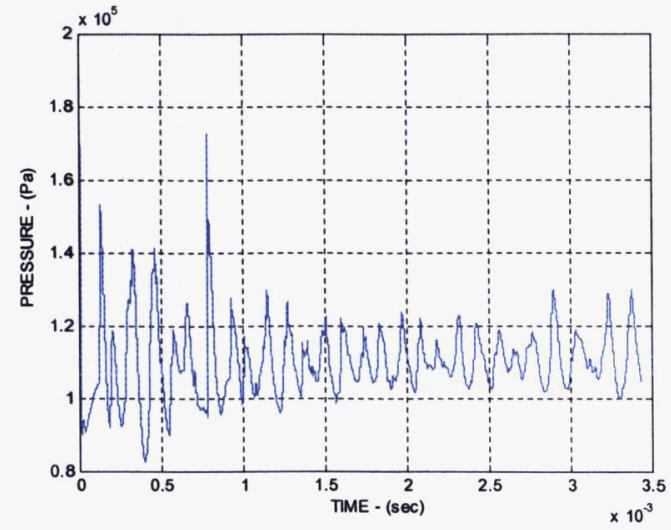

(a)

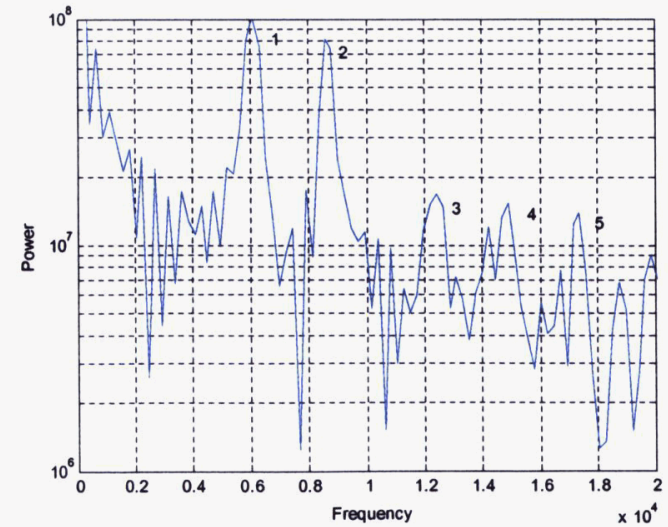

(b)

Figure 5: (a) Time history of the pressure of a lean-premixed flame in a two-dimensional axisymmetric geometry and (b) the FFT of the corresponding pressure data 


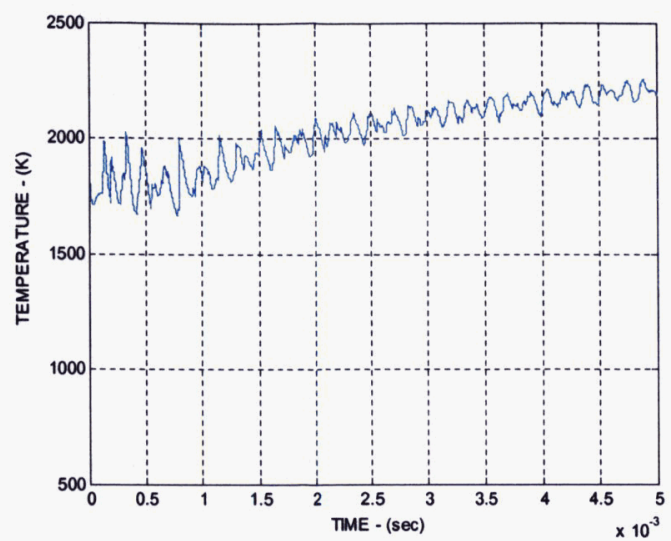

Figure 6: Time history of the temperature of a lean-premixed flame in a two-dimensional Cartesian geometry

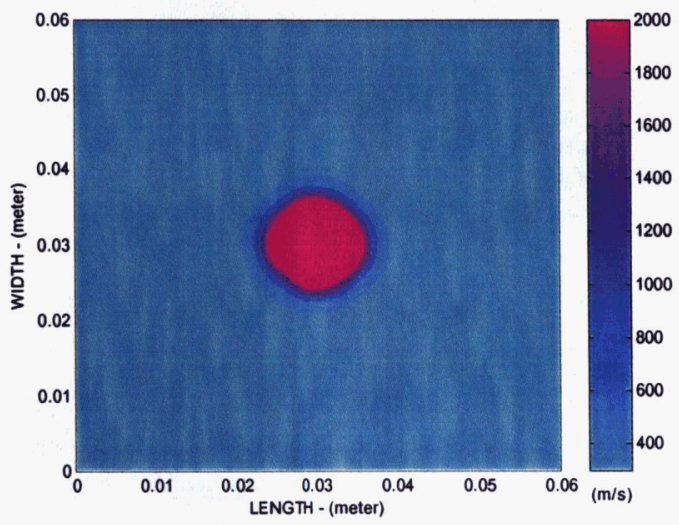

Figure 7: Instantaneous temperature contours of a premixed-flame with no acoustic disturbance at $\mathrm{t}=0.005$ seconds for the Cartesian geometry

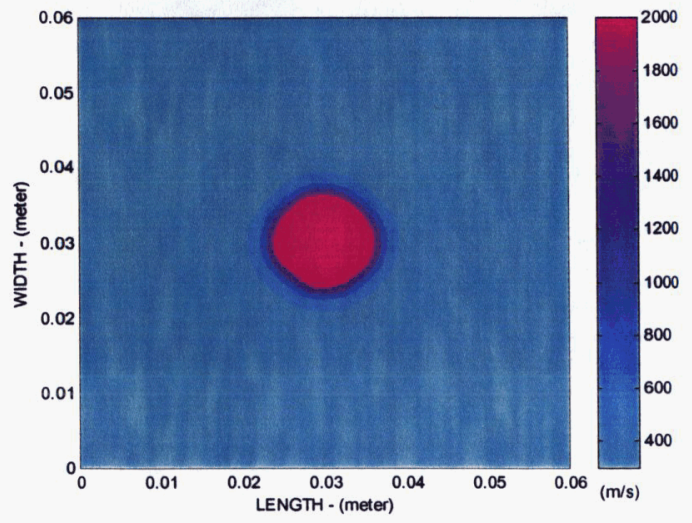

(a)

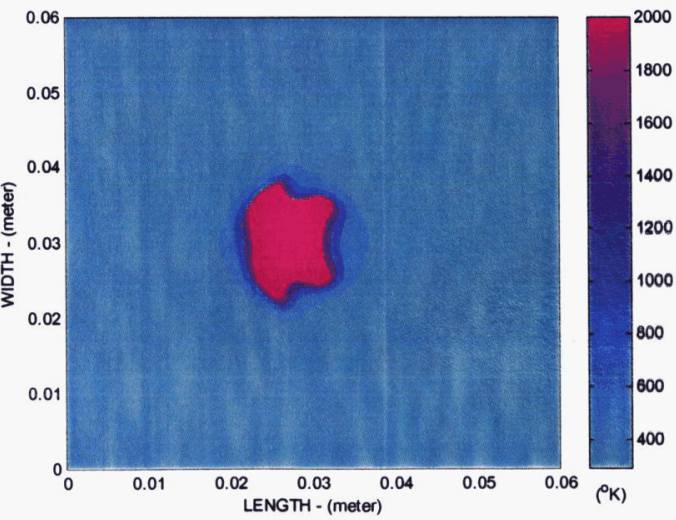

(b) 


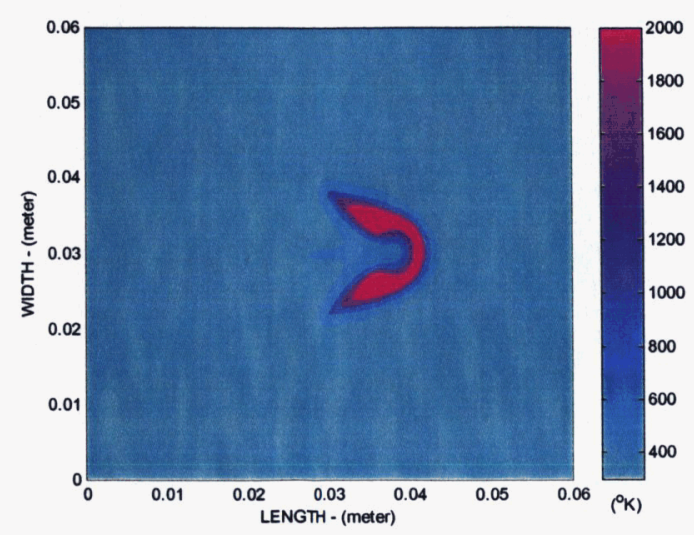

(c)

Figure 8: Instantaneous temperature contours of a combustion-acoustic interaction in a 2-D Cartesian system at $\mathrm{t}=0.005$ seconds for $(a) \varepsilon=0$, (b) $\varepsilon=0.3$ and (c) $\varepsilon=0.6$.

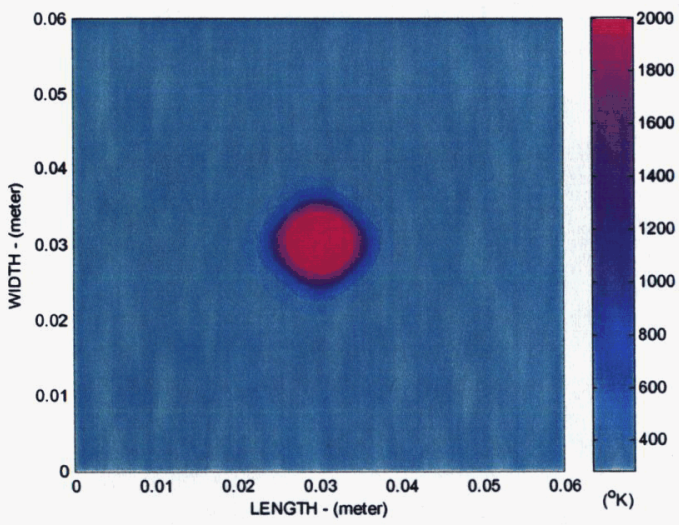

(a)

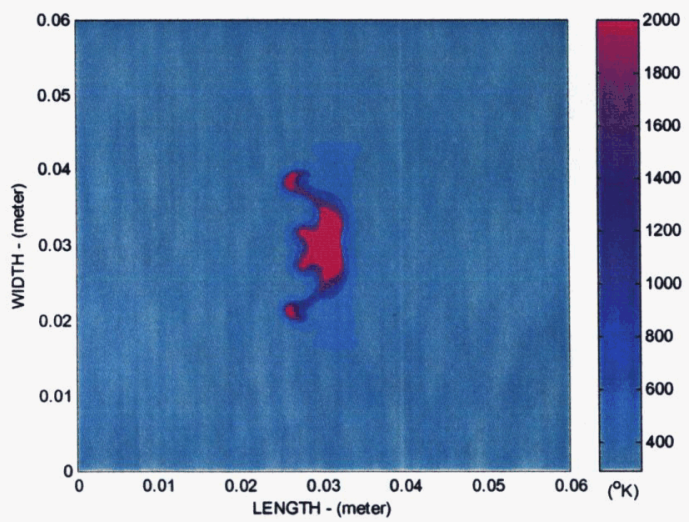

(b)

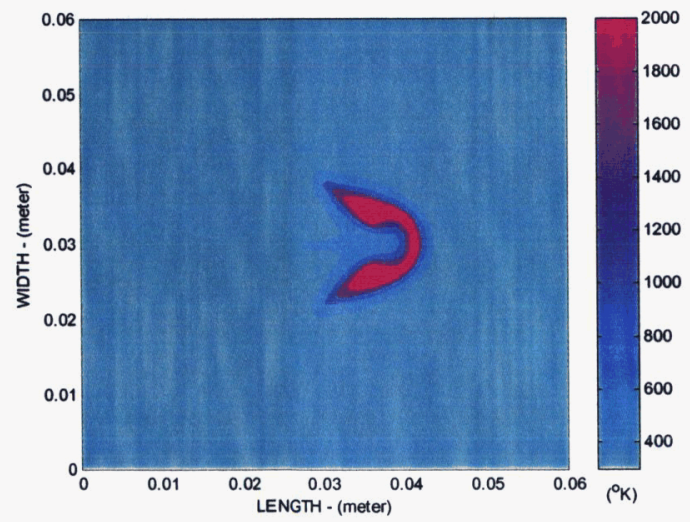

(c)

Figure 9 - Instantaneous temperature contours of a combustion-acoustic interaction in a 2-D Cartesian system at $\mathrm{t}=\mathbf{0 . 0 0 2 5}$ seconds for (a) no-disturbance, (b) off-resonant and (c) resonant case 
GRID
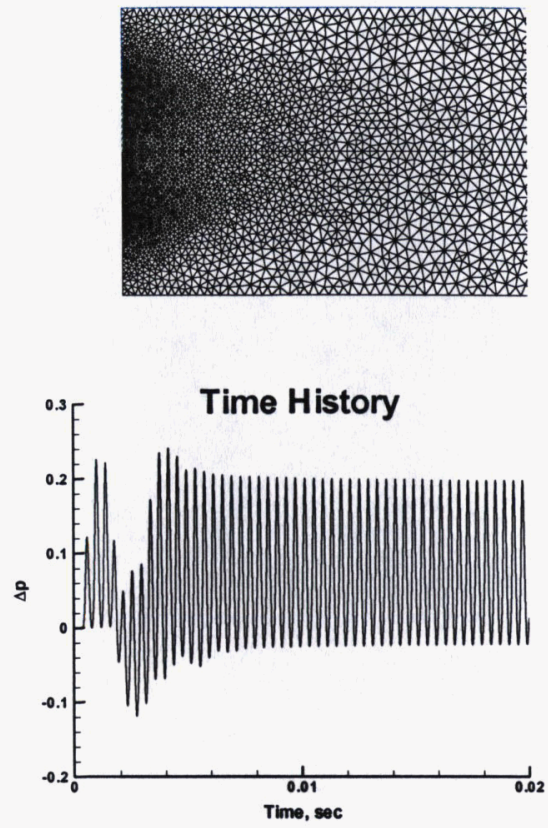

Pressure Contours
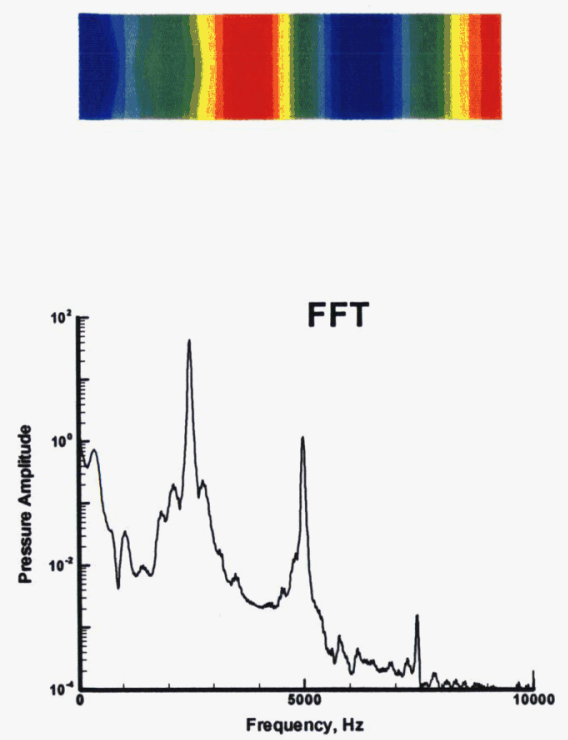

Figure 10: Resonant acoustic wave interaction with a combustion chamber mode.

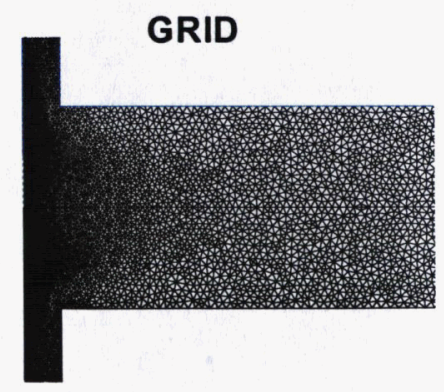

Pressure Contours
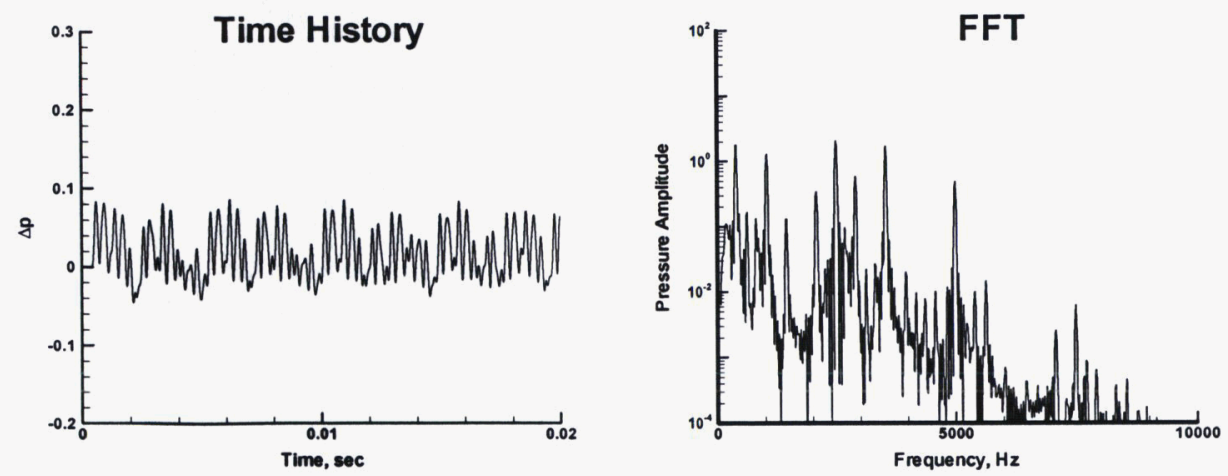

Figure 11: Effect of a quarter-wave on the resonant interaction between an acoustic wave and a chamber mode. 


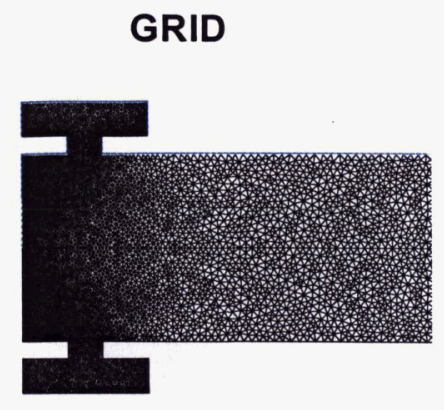

\section{Pressure Contours}
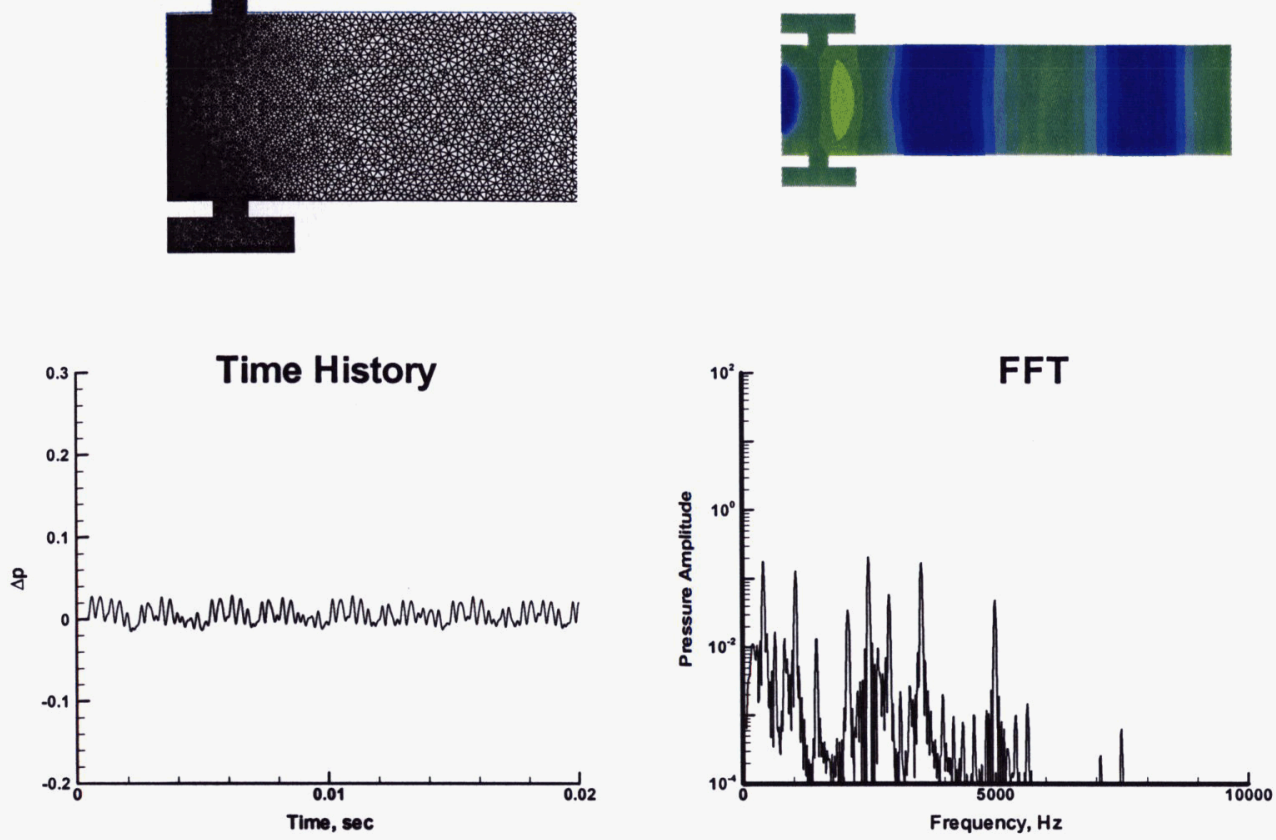

Figure 12: Effect of an acoustic cavity on the resonant interaction between an acoustic wave and a chamber mode. 